\title{
Linx
}

Revue des linguistes de l'université Paris X Nanterre

$12 \mid 2002$

«Comme la lettre dit la vie »

\section{Les leçons du corps et du rire dans les Cent Nouvelles Nouvelles}

Jean Dufournet

\section{OpenEdition}

Journals

Édition électronique

URL : http://journals.openedition.org/linx/1282

DOI : 10.4000/linx.1282

ISSN : 2118-9692

Éditeur

Presses universitaires de Paris Nanterre

\section{Édition imprimée}

Date de publication : 1 octobre 2002

Pagination : 80-85

ISSN : 0246-8743

\section{Référence électronique}

Jean Dufournet, "Les leçons du corps et du rire dans les Cent Nouvelles Nouvelles », Linx [En ligne],

12 | 2002, mis en ligne le 10 octobre 2012, consulté le 01 mai 2019. URL : http://

journals.openedition.org/linx/1282 ; DOI : 10.4000/linx.1282

Ce document a été généré automatiquement le 1 mai 2019.

Département de Sciences du langage, Université Paris Ouest 


\title{
Les leçons du corps et du rire dans les Cent Nouvelles Nouvelles
}

\author{
Jean Dufournet
}

1 Il est dans les Cent Nouvelles Nouvelles deux domaines où s'effacent toutes les différences sociales. Celui de la tromperie d'abord. L'Ecossais de la N.45 berne, grâce à son travestissement en lavandière, toute la ville de Rome, jusqu'aux plus riches personnages. Mais, s'il peut faire fi des différences sociales, c'est que l'appétit sexuel les nivelle. Les cordeliers d'Ostelleric en Catalogne (Hostalric) prélèvent leur dîme sur toutes les couches de la société :

«Les pouvres simples femmes, qui mieulx cuidoient ces bons freres estre anges que hommes terriens, ne refuserent pas ce disme a paier. Il n'y eut celle qui ne le paya a son tour, de la plus haulte jusques a la maindre; mesme la dame du seigneur n'en fust pas excusee. Ainsi furent toutes les femmes de la ville appaties a ces vaillans moynes. Et n'y avoit celluy d'eulz qui n'eust a sa part de XV a XVI femmes le disme a recevoir. Et a ceste occasion Dieu scet les presens qu'ilz avoient d'elles, tout soubz umbre de devocion. » (N.32, p. 216-217, 1. 55-65) ${ }^{1}$

Dans la N.3, par symétrie des deux histoires amoureuses entre le seigneur et la meunière comme entre le meunier et la dame, on assiste à une sorte de réciprocité qui abolit toute classe sociale, puisque le meunier devient le rival du seigneur qu'il fait cocu et qui l'implore de garder le secret sur toute cette aventure.

Plus profondément, ces exemples et bien d'autres indiquent que le monde des Cent Nouvelles Nouvelles est centré sur le corps, comme le montre l'expression métaphorique de l'acte sexuel, comparé à la plupart des champs de l'activité humaine, ainsi qu'en témoigne un très rapide échantillonnage ${ }^{2}$.

- La guerre et le combat courtois :

« ... et s'en alla combattre ou lit de sa dame la chambriere tout prest pour son veu accomplir, ou qu'il fut bien receu et rencontré. Et tant rompirent de lances qu'ilz furent si las et recreuz ... »

(N.59, p. 366, 1. 44-47);

« ... quant il oyt le tamburch et noise des combatans ...» (N.87, p. 505, 1. 96-97);

«...par plusieurs foiz, avant et puis le partement de son amy, luy avoit presenté la bataille

(...) Car, s'il avoit bonne volunté de combatre et faire armes, elle n'avoit pas mains de desir 
de luy de lyer son emprinse et le fournir de tout ce qu'il la vouldra requerre » (N.22, p. 146, 1. 34-36 et 46-49). expressions qui, désignant à l'origine un alibi commode, finissent par désigner l'acte sexuel comme aller en pelerinage ou à matines. Le maistre curé de la N. 64 «faisoit rage de confesser ses parrochiennes. De fait, il n'en eschappoit pas une qui ne passast par la, voire des plus jeunes. Au regard des veilles, il n'en tenoit compte » (p. 402, l. 7-10). La femme de la N.38, qui est un fin cordon bleu, « aux Cordeliers s'en va ... pour menger la lemproye et gaigner les pardons, comme assez avoit de coustume » (p. 264, 1. 112-114).

Sans doute le narrateur se délecte-t-il à multiplier les métaphores, qui sont souvent savoureuses; mais surtout il tient à choisir divers pans de la condition humaine pour désigner l'acte sexuel, en sorte que le corps et ses plaisirs deviennent le foyer d'où rayonnent toutes les activités de l'homme. En fait, c'est moins l'acte sexuel que la liberté du corps qui constitue le point central du recueil. La diversité métaphorique a pour but de manifester la foisonnante richesse du corps dont la langue s'épuise à rendre la généreuse abondance.

12 Parmi toutes ces liaisons que le corps établit, il en est deux qui requièrent plus particulièrement notre attention, et d'abord celle qui rapproche l'amour physique de la 
nourriture. Si l'amour est une thérapeutique efficace, il l'est d'autant plus qu'il accompagne un autre besoin fondamental, celui de se nourrir. Sans doute cette comparaison produit-elle à son tour des jeux de mots savoureux, comme dans la N.83 où le trait d'esprit d'un carme glouton conclut un repas pantagruélique décrit sur plus de cinquante lignes :

«Je croy (dit la servante) que, si vous eussez esté l'un de ceulx qui la furent repeuz (lors du miracle de la multiplication des pains), qu'on n'en eust point rapporté de relief, car vous eussez bien tout mengé, et moy aussi se je y eusse esté! - Vrayement, m'amye, dit le moyne, qui estoit ung garin tout fait, je ne vous eusse point mengee, mais je vous eusse bien embrochée et mise en rost, ainsi que vous pensez qu'on fait. » (p. 488, 1. 94-101).

La ripaille sert souvent de cadre à l'acte sexuel qui est comme le contrepoint d'un monde d'abondance, comme dans la N.35 où la chambre du chevalier étranger estoit belle a bon escient, bien mise a point; et estoit le beau buffet fourny d'espices, de confictures et de bon vin de plusieurs façons. (p. 249, 1. 110-113). Les métaphores relatives à la nourriture témoignent toutes de l'abondance vitale dont le corps est le foyer. La N.38, à partir d'un schéma narratif proche de celui du fabliau des Tresses ${ }^{3}$ est construite sur la répétition du mot lamproie qui, d'abord employé au sens propre (une belle et grosse lemproye, telle belle lemproie, etc...) devient peu à peu équivoque : il s'agit dès lors pour la femme d'un marchand de Tours de menger de la lamproie avec un frère cordelier. La maitresse de maison manifeste de singuliers talents de fine cuisinière et d'amoureuse experte, qu'elle relie elle-même dans une réplique à son mari : "... il me souvient tres bien que aujourd'uy, au matin, vous feistes de tres bon appetit le jeu d'amours» (p. 266, 1. 169-170). Dans la N.17, un grand clerc chevalier « ne jeunoit jour de l'amoureux mestier tant qu'il trouvast rencontre » (p. $115,1.15-16)$, et la jeune femme de la N.92 affirme « qu'elle ne se partiroit point qu'elle n'eust encores ung picotin» (p. 522, 1. 51-52). Cette équivalence entre l'amour et la nourriture se retrouve dans la description de l'acte sexuel. La N.87 fait état dans l'étreinte d'une dévoration symbolique: le chevalier trompé «vit les deux amoureux qui se demenoient tellement l'un contre l'autre qu'il sembloit qu'ilz deussent menger l'un l'autre, tant mettoient et joindoient leurs dens ensemble » (p. 505, 1. 98-102). A l'occasion, c'est par cette équivalence qu'un gentilhomme volage justifie qu'il trompe sa femme, pourtant très belle :

\footnotetext{
«Et tu pourrais admettre que j'échappe à l'ennui alors que tu veux que je me contente du seul corps de ma femme! Tu peux bien t'imaginer, par ma foi, que j'en suis aussi rassasié que tu l'es, toi, de tes pâtés et que je pourrais avoir envie de varier les plaisirs avec une autre femme, quand bien même je ne l'aimerais pas autant que la mienne, tout aussi volontiers que toi avec une autre nourriture que tu n'aimes pas autant que les pâtés. " (N.10, p. 83, 1 . 134-141) ${ }^{4}$
}

Ces comparaisons, loin de se borner à faire la part des besoins du corps ou à en expliquer la lassitude, l'exaltent comme le lieu de l'abondance et du plaisir.

L'autre liaison métaphorique, qui nous semble significative, ressortit davantage à la littérature: elle établit une relation entre le corps et le livre, entre l'acte sexuel et l'écriture. L'on sait que l'assimilation de l'acte sexuel à la parole est on ne peut plus fréquente, souvent rapide par le seul emploi des mots devise et deviser, et parfois plus explicite, comme dans la N.67: "Ce maistre chaperon fourré fit tant, par moyens et d'argent et aultrement, qu'il parla a la belle cordoaniere dessoubz sa robe et a part » ( . 414, 1.16-18) et encore plus dans la N.71 : 
"Quand ce gentil chevalier vit son hostesse preste d'oyr, d'entendre et escouter ce qu'il vouldroit dire, pensez qu'il fut joyeux outre mesure (...) et commença sa harengue a l'heure ... (p. 432, 1. 27-30 et 34-35) elle, vous ne serez pas en mon livre enregistré n'escript, que premier ne passez a monstre et que je ne sache quel harnois vous portez » (p. 107, 1. 86-89). Elle se développe en des équivoques tout à fait claires dans la N.23. La femme d'un procureur se livre à toutes sortes de provocations envers un clerc de son époux dont elle s'est enamourée et qui semble rétif à ses avances. Aussi, tandis qu'il écrit, tout occupé par son travail de secrétaire, «mesmes pour le plus empescher et baillier destourbier, elle respandit sur buffet, sur papier et sur sa robe, son cornet a l'encre ». Le clerc, qui finit par comprendre, répond à son invite en se masquant derrière son travail d'écriture : «Pour ce, dit il, que vous avez respandu mon cornet a l'encre et avez brouillé et mon escripture et ma robe, je vous pourray bien brouiller vostre parchemin; et affin que faulte d'encre ne m'empesche d'escripre, je pourray bien pescher en vostre escriptoire». (p. 152, 1.53-58). Le sexe féminin est progressivement assimilé à l'objet sur lequel on écrit et relié au travail de l'écriture dans son sens technique. A partir de cette association, le recueil construit un projet cohérent. Le corps, plus précisément le «bas corporel », est mis en rapport avec le livre, et certaines nouvelles esquissent en filigrane la comparaison entre le savoir livresque et le savoir du corps. Dans la N.17, la position sociale et la science du mari, que sa femme a surpris en flagrant délit d'adultère, cèdent le pas à toute-puissance du corps, ce qui promeut dans la joie le caractère irrésistible de certains besoins physiologiques, comme le manifeste ce savoureux dialogue entre la femme et le mari : «Et quand elle le voit en cest estat, et affublé du buleteau (blutoir), elle luy va dire : « Ha ! monseigneur, et qu'est cecy? et ou sont voz lectres, voz grands honeurs, voz sciences et discrecions? » Et monseigneur, qui deceu se voit, respondit tout subitement: « Au bout de mon vit, dame, la ay je tout amassé aujourd'uy » (p. 119, 1. 132 - 138). Le corps, par sa bonne santé et sa prodigue toute-puissance, déçoit toute science livresque. Telle est la leçon de la N. 37 où le mari jaloux, en dépit de l'immense savoir qu'il a accumulé sur les ruses et tromperies des femmes, est inéluctablement trompé. La faillite du livre face aux pouvoirs insaisissables du corps qui se confondent avec ceux de la femme, est alors éclatante :

«Est ce cela? dit il. Nostre Dame, ce tour n'estoit pas en mon livre! Allez, allez, je voy bien que c'est. " Il eust voluntiers dit qu'il estoit coux. Et creez que si estoit il a ceste heure, et ne l'en sceut oncques garder livre ne brevet ou pluseurs tours estoient enregistrez » (p. 260, l. 172-177) rassembler des nouvelles qui tirent du corps lui-même abondance et vigueur. Sans doute faut-il voir, derrière un jeu métaphorique exubérant, derrière cette prédilection constante pour les "jeux d'amour», le projet véritable de l'ouvrage: la célébration joyeuse de la puissance inépuisable du corps, principe de vie et d'écriture.

18 En effet, les Cent Nouvelles Nouvelles tendent à en exalter les ressources. S'il existe des cas d'impuissance, témoin les N.28 et 35, ces rares exceptions sont vite oubliées, eu égard aux prouesses que réalisent les personnages, et que le narrateur dénombre : «Lors de rechef la baise et accole, et ne la souffrit partir tant qu'il eut besoigné deux foiz, qui gueres ne luy cousterent, car alors il estoit frez et jeune et homme fort a cela» (N.39, p. 270, 1 . 69-72); « ...et disoit, affermoit et juroit sur son honneur qu'il portoit le plus beau membre, le plus gros et le plus quarré qui fust en toute la marche d'environ; et avecques ce, et qui n'empire pas le jeu, il s'en aidoit tellement et si bien que les quatre, les V, les VI 
foiz ne luy coustoient non plus que s'on les prinst en la corne de son chaperon » (N.65, p. 407, 1. 10-16). Même le vieillard, marié à une jeune fille, est à la hauteur de la situation (N. 53, p. 341, 1. 100-101). Dans ce monde de ruse et de déception, où il n'est de confiance que pour être trompée, seul le corps tient ses promesses, même quand le héros, un berger, émet des propos qui peuvent passer pour des rodomontades :

«Et le bon bergier, qui n'estoit fol ne esperdu, leur dit que par la mort bieu il oseroit bien emprendre de faire la besoigne VIII ou IX foiz pour nuyt. (...) Il monstra de fait ce dont il s'estoit vanté de bouche. Car avant que le jour venist, il fist tant que le cerf eut VIII cornes accomplies, laquelle chose sa dame print bien en gré » (N.57, p. 358, 1. 32-35 et 49-52).

L'effet de redondance, produit par ces nouvelles, finit par convaincre que le corps illumine de sa loyauté et de son allégresse le recueil tout entier.

Cette leçon du corps va de pair, comme chez Villon ${ }^{5}$, avec la prise de conscience de la mort dans toute sa cruauté, puisque, par deux fois au moins, surgit l'image du pourrissement. Mais le corps, en s'adonnant à l'amour physique, peut dépasser cette condition mortelle : «Et le bon clerc incontinent fault a sa garde, et, le plus longuement que il et sa dame oserent, n'espergnerent pas les membres qui en terre pourriront » (N.13, p. 96, l. 165-168). Dans la N.55, c'est la vision la plus crue de la mort qui stimule l'appétit sexuel de la jeune fille atteinte de la peste. Elle confie à sa voisine : « ...j'ay grand regret que force m'est aujourd'huy de habandonner ce monde et les beaulx et bons passetemps que j'ay euz longtemps ; mais encores, par mon serment, a dire entre vous et moy, mon plus grand regret si est qu'il fault que je meure avant que savoir et sentir des biens de ce monde. Telz et telz m'ont maintesfoiz priee, et si les ay refusez tout plainement, dont me desplaist » (p. 347, 1. 16-22). Elle guérit effectivement par la « compagnie » de plusieurs jeunes gens auxquels elle transmet le mal dont ils meurent. Telle est la conclusion de la nouvelle, ironique mais lourde de sens : «Et disent les maistres qu'elle eschappa de mort a cause d'avoir senty des biens de ce monde. Qui est notable et veritable exemple a plusieurs jeunes filles de point refuser ung bien quand il leur vient» (p. 351, 1. 123-126). L'amour physique est un moyen d'exorciser la peur naturelle de la mort, comme, symboliquement, la mise à distance qu'opère un compaignon a demy fol, au pied du gibet, par une chanson qui lui sauva la vie (N.75, 1. 84-101).

21 Ailleurs, un jeune homme s'amuse de l'annonce de la mort de sa mère qui se rétablit toujours in extremis. Et l'auteur de jouer subtilement du comique de répétition.

«Dictes vous donc, dit il (le fils), qu'elle s'en va? Par ma foy, je ne l'ose croire: tousjours dit elle ainsi, mais rien n'en fait. - Nenny, nenny, dirent ses gardes, c'est a bon escient; venez vous en, car on voit bien qu'elle s'en va. - Je vous diray, dist il ; allez devant et je vous syeuz; et dictes bien a ma mere, puis qu'elle s'en veult aller, que par Douay point ne s'en aille, car le chemin est trop mauvais. » (N.77, p. 459).

Mais le fils paiera de sa vie son insolence, puisque, sa mère ayant fini par mourir, «tant fort mesmes le print il au cueur que devant n'en tenoit compte par semblant, que au bout de XV jours de dueil il en mourut » (p. 460, 1. 81-83). Cette fin témoigne de l'ambiguïté de tout exorcisme comme de tout le recueil : mise à distance, la mort garde sa redoutable puissance.

Dans la N.6, « un grand lourd Hollandois si tresyvre que merveilles » exige d'être confessé par un prieur augustin qu'il a rencontré ; puis il veut qu'il le tue pour qu'il aille tout droit en paradis. Il finit par s'écrouler par terre: «Mais vous n'avez garde qu'il se relieve, mesmes cuide vrayement estre en paradis » (p. 63, 1. 87-89). On a beau l'appeler, il ne 
cesse de répéter qu'il est mort, en une scène qui rappelle, en moins brillant, la fausse mort de Pathelin :
«Laissez moy, laissez, je suis mort. - Non estes, non, dirent ses compaignons; il vous en fault venir avecques nous. - Non feray, dist l'yvroigne, ou yrois je? Je suis mort et desja en paradis. - Vous vous en viendrez, dirent les aultres : il nous fault aller boire. - Boire! dit l'aultre; jamais ne buray, car je suis mort. » Quelque chose que ses compaignons lui deissent ne fissent, il ne vouloit partir ne mettre hors de sa teste qu'il ne fust mort. » (p. 63-64, l. 108-117).

La parole et l'écriture permettent d'exorciser la peur de la mort; elles disposent d'un indubitable pouvoir de subversion qui transcende les limites de la condition humaine.

Liée à cette première leçon, il en est une autre qui s'affirme contre les "ploreries » de toute sorte : celle du rire. Certes, la douleur peut s'exprimer en termes hyperboliques qui ne le cèdent en rien à la tradition épique et romanesque, comme dans la N.69 où une femme, qui s'est remariée, apprend le retour de son mari fait prisonnier à la bataille de Nicopolis :

«Et ces parolles dictes, accompagnees de grands larmes, son treshoneste, tresvertueux et loyal cueur s'evanuyt, et cheut paulmee. Elle fut prinse et portee sur ung lit, et luy revint le cuer. Mais depuis ne fut en puissance d'homme ne de femme de la faire menger ne dormir, ainçois fut trois jours continuelz tousjours plorant, en la plus grand tristesse de cueur que jamais femme fut ...Et tost aprés elle mourut, dont ce fut tresgrand dommage » (p. 425, 1. 85-97)

Nous avons vu plus haut la fin d'un fils qui trop longtemps prit à la légère la mort de sa mère.

Mais cette douleur est vite tournée en dérision par la parodie. Ou bien elle débouche sur l'obscénité et la scatologie, comme dans le cas de cette pouvre fille qui souffre d'une maladie «que communement l'on appelle broches » (N.2, p. 32, 1. 27-28), c'est-à-dire des hémorrhoïdes. Après qu'on eut fait en vain appel à une matrone et à de nombreux médecins qui jamais ne virent tel cas « en leurs livres », le manège d'un cordelier borgne entraine chez la jeune patiente une réaction inattendue : elle essaie de se retenir de rire, "Mais si mal, hélas! luy advint que ce ris a force retenu fut converty en ung sonnet dont le vent retourna si tres a point la pouldre que la pluspart il fist voler contre le visaige et l'œil de ce bon cordelier, lequel, sentent ceste douleur, habandonna tantost et vaissel et tuyau, et a peu qu'il ne cheut a la renverse, tant fut fort effrayé » (p. 35, 1. 128-135). Ou bien l'expression de la douleur relève de la comédie plus ou moins réussie d'un clerc qui abuse son maître, "car grosses larmes, en parlant, luy descendoient en tresgrand abundance; et n'est homme qui ne cuidast qu'elles ne fussent ou de contrition, de pitié ou des tresbonne entencion» (N.13, p. 93, 1. 66-70), ou encore d'un président de Parlement qui a fomenté la mort de sa femme : « Le bon president, plus joyeux en cueur qu'oncques triste ne fut, se monstra tresdesplaisant; et de fait se laissa cheoir du hault de luy, menant trespiteux dueil en regretant sa bonne femme. Il maudissoit sa mule, les beles nopces qui firent sa femme partir ce jour» (N.74, p. 313, l. 114-119). Parfois, la douleur, quelque extrême qu'elle soit, se calme rapidement : «Au fort, el s'appaisa, puisque aultre chose estre n'en peut. Et quant vint environ ung mois après le partement de son amy, desir luy eschaufa le cueur et si luy vint ramantevoir les plaisans passetemps qu'elle souloit avoir...» (N.22, p. 145-146, 1. 25-30). Ou encore la description hyperbolique du chagrin tourne vite à la mascarade, qu'il s'agisse d'une religieuse qui ne peut se résoudre à avoir "compaignie d'un homme" malgré les recommandations pressantes de son médecin (N.21), ou d'un jeune marié qui s'afflige de ne pas avoir « ronciné » suffisamment ses parents pour allonger leurs vies comme il l'a fait pour sa femme, et dont le ridicule 
éclate, puisque « il n'y eut celuy de la table aprés ces motz a pou qui se tenist de rire, mais non pourtant il s'en garda qui peut » (N.20, p. 138, 1. 225-227).

Ce sont ces "ploreries" que dénonce la bonne humeur dans mainte nouvelle dont certaines sont rythmées par le rire, telle que la N.27, qui raconte comment une dame met hors d'état de nuire "son bon mary, tenant le lieu en ce cas du tresmaudit Dangier» (p. 182, 1. 21-22), en l'enfermant dans un bahut: «... et tout jouant, riant et esbatant, prindrent toutes ensemble homme et bahu ...» (l.104-105); «Il (l'ami) fut receu joyeusement et lyement ...» (1. 124-125); « ...quand toutes ces femmes entrerent dedans, qui si tresfort rioyent, et de si grand cueur, qu'elles ne sceurent mot dire grand piece aprés. Et monseigneur, qui devoit faire merveilles, quand il les vit rire en ce point, ne se peut tenir de les contrefaire. Et madamoiselle, pour luy faire compaignie, ne s'i faignoit point. La veissiez une merveilleuse risée, et d'un costé et d'aultre, mais celuy qui en avoit le mains cause ne s'en pouvoit ravoir » (1. 207-216); « ... et madamoiselle et ses femmes le suyvent, qui faisoient de luy, je vous asseure, grans risees; et creez que la messe ne se passa pas sans foison de ris soudains, quand il leur souvenoit du giste que monseigneur a fait ou bahu ...» (1. 248-252).

Du recueil il ressort que le rire possède une parenté certaine avec le corps et ses plaisirs. Comme l'amour, il libère une abondance, jusque-là contenue, qui éclate. Les nouvelles explorent cette parenté. Dans la N.54, les rires de la jeune fille sont autant de provocations à l'adresse d'un charreton «qui estoit ung tresbeau compaignon, fort et viste » et à qui, de surcroît, elle jette des coussins qui le font tomber :

"... elle luy gecta ung coussin sur la teste, et le fist cheoir a pates, et puis commença a rire tresfort et bien hault. Le charreton se sourdit et la regarda rire, et dist: "Par Dieu, madamoiselle, vous m'avez fait cheoir mais creez que je m'en vengeray bien, car avant qu'il soit nuyt je vous feraytumber. - Vous n'estes pas si mal gracieux ", dist elle. Et, en ce disant, elle prend ung aultre coussin, que le charreton ne s'en donnoit garde, et le fait arriere cheoir comme avant. Et s'elle risit fort auparavant, elle ne s'en faindit pas à ceste heure» (p. 344, 1. 38-49).

Le rire intervient souvent dans le cadre de la ripaille, joyeux affranchissement du corps comme lieu d'abondance. S'il accompagne la satisfaction du corps, il a, tout autant que l'amour physique, un effet salutaire et il instaure entre les gens une communauté heureuse. Dans la N.83, le rire, qui est communicatif, libère les cœurs et les corps, apaisant toute réaction de colère et de hargne : si le valet et la chambrière sont choqués par la voracité et l'inconduite d'un religieux glouton, le rire a raison de leurs réticences, puisque « la dame commença a rire, et si firent le varlet et la chambriere, malgré qu'ilz en eussent » (p. 488, 1. 101-103). Et le recueil d'insister sur son aspect thérapeutique : « Et qui a ceste heure l'eust veu rire, jamais n'eust eu les fievres » (N.14, p. 158, 1. 129-130); « qui adonc veist rire le varlet et la meschine, il n'eust jamais eu les fievres!» (N.38, p. 487, l. 76-77). En tout état de cause, il permet de retrouver une spontanéité que les Cent Nouvelles Nouvelles se plaisent à mettre en scène.

31 Toutefois le rire peut se retourner contre celui qui le manie imprudemment. C'est le cas de ce père qui, "pour faire rire la compaignie ", s'obstine, dans la N.66, à s'enquérir auprès de son fils de « l'estat et gouvernement de celles qui estoient aux estuves avecques sa mere » (p. 412, 1. 19-21) et qui finit par obtenir de lui une réponse naïvement grivoise aux dépens de sa mère :

"Et nous commenceasmes tous a rire et a boire d'autant, et parler de cest enfant qui caquetoit si bien. Mais sa mere n'en savoit sa contenance, tant estoit honteuse, pour ce que son filz avoit parlé du nez; et croy bien, depuis il en fut tresbien torché, car il avoit encusé le 
secret de l'escole. Nostre hoste fist du bon compaignon; mais il se repentit assez depuis

d'avoir fait la question, dont la solution le fist rougir » (p. 413, 1. 49-57)

32 La leçon de la nouvelle, dans ce combat constant du recueil contre toute illusion de possession, est qu'on ne s'approprie pas facilement le rire, insaisissable, parce que, produit de la spontanéité, il est lui aussi le lieu d'une ambiguïté extrême dont les nouvelles illustrent le caractère irréductible.

Tantôt il réconcilie, et le texte s'achève dans une saine bonhomie qui libère l'allégresse du corps: les trompés pardonnent aux trompeurs, les cocus bénissent leurs rivaux ou concluent la paix sans plus discuter, comme si la force du rire était précisément dans cette capacité d'apaisement : « tous ceux qui oyrent ceste response commencerent a rire de grand cueur et dirent bien qu'il estoit bon homme. Si s'efforcerent a ceste occasion de faire sa paix avec son pere. Et tant s'i employerent qu'ilz en vindrent au bout, et fut tout pardonné d'un costé et d'aultre » (N.50, p. 326, 1. 57-62) ; « Le cyrurgien, qui estoit le plus gentil compaignon et des aultres le meilleur homme, commença a rire, et firent la paix. Et croy bien que tous deux, quand l'œil fut gary, s'accorderent a besoigner par terme » (N.87, p. 506, 1. 111-114). Les plus grands courroux ne résistent pas au rire, même s'agissant d'un viol : "Pensez qu'il y eut grand risee, après la conclusion de ce procés, de ceulx de la justice et de tous les assistens » (N.25, p. 161, 1.78-80). Le mari berné a une réaction identique dans la N.73 quand il découvre, enfermé dans un casier, son rival « doré et empapiné d'oeufz, de fromaige, de laict et aultres choses plus de cent. Ce pouvre amoureux estoit tant piteusement appoincté qu'on ne savoit du quel il avoit le plus. Et quand le bon mary le vit en ce point, il ne se peut tenir de rire, combien que courroussé deust estre » (p. 446, 1. 210-215).

Tantôt le rire châtie et ne laisse derrière lui qu'une grimaçante mascarade. Rire de dérision qui n'est pas dupe du mensonge d'un mari que sa femme a découvert avec sa chambrière :

"Il y eut alors de grands éclats de rire chez ceux qui étaient réunis là, et sans pitié ils s'acharnèrent sur le mari jusqu'à l'hallali. La dame, en effet, leur raconta comment il s'était fait passer pour malade et toute la comédie qu'il avait jouée ... » ${ }^{6}$

De même, un âne, de son braiement, nargue un jaloux qui croyait tenir son rival : «Et, ainsi que le bon homme eut ouvert la huche et que cest asne veist la lumiere, il commença a ricaner si hideusement qu'il n'y eut la si hardy qui ne perdist sens et memoire. Et quand ilz virent que c'estoit ung asne et qu'il les avoit ainsi abusez, ilz se vouldrent prendre au marchant ...» (N.61, p. 383-384, 1. 177-183). Dans la N.2, le rire, par un enchaînement farcesque, provoque le châtiment du frère cordelier qui, au bout du compte, y perd son œil valide (N.2, p. 35, 1. 123-141).

Par cette oscillation entre la bonhomie et le châtiment, le rire participe de ce grand mouvement de relativisation qui parcourt l'ensemble des Cent Nouvelles Nouvelles dont le sens reste insaisissable, comme celui de ces histoires qui peuplent le livre et comme la voix du narrateur qui les rapporte.

De cette vision du monde, il reste une leçon d'allégresse et de libération dont le corps est le foyer par son exubérance et ses " combats d'armes ", et tout autant, à son image, le livre lui-même, foisonnant, aussi inépuisable que le «bas corporel » et que la tromperie féminine, et dont la dernière nouvelle est emblématique, puisqu'elle se poursuit au-delà de la fin du recueil : 
"Le maître d'hôtel et tous les autres serviteurs se mirent à rire et feignirent d'entrer dans le jeu de leur maître en gobant ce mensonge subtil et paré de si belles couleurs. Ils y trouvèrent par la suite une bonne raison d'apprécier leur maître, ainsi qu'une bien bonne histoire à raconter en maintes occasions, ici ou là. " si le texte se donne à lire entre les textes, si son sens moral ne se laisse pas saisir en luimême, pas plus que chaque récit, mais doit s'envisager dans l'exercice de sa dérobade et la faillite subversive de l'univoque et de l'exemplaire, cette dégradation d'un monde en fuite généralisée et perpétuelle conduit à la célébration du corps et du rire, exorcisant la peur de la mort et ramenant la lecture à sa jubilation première.

éclate la richesse d'un monde dérobé où se perd la voix du narrateur et où le sens littéral ou moral se disperse dans la duplicité et la fuite. Rien n'y appartient à personne, ni la femme au mari, ni l'argent aux avares, ni le sens final du péché aux prédicateurs. Ce monde dérobé esquive tout achèvement dans l'inépuisable floraison des récits et des corps, dans la multiplicité des détours et des cachettes, des escaliers et des portes qui permettent aux amants de s'échapper.

C'est ce monde dissimulé aux regards que le narrateur invite à explorer dans ses recoins et qu'il faut découvrir par une porte dérobée du texte dont la quête en perpétuel devenir a plus de saveur que sa découverte: « chose eue en dangier est trop plus cher tenue que celle qu'on a a bandon » (N.57, p. 415, 1. 47-49).

\section{NOTES}

1. Nous citerons les Cent Nouvelles Nouvelles d'après l'édition de Franklin P. Sweetser, Paris, Minard et Genève, Droz, 1966, Textes littéraires français, 127). Nous indiquerons successivement le numéro de la nouvelle (par ex. N.32), la page et les lignes citées.

2. Nous nous bornerons à un rapide relevé pour montrer l'éventail du jeu métaphorique. Une présentation complète demanderait un long article.

3. Qu'on pourra lire, par exemple, dans notre édition bilingue, Fabliaux du Moyen Age, Paris, GF Flammarion, 1998, p. 289-311.

4. Traduction de Roger Dubuis, (1991), Les Cent Nouvelles Nouvelles, Lyon, Presses Universitaires de Lyon, pp. 65-66.

5. Voir notre étude « Deux poètes du Moyen Age face à la mort: Rutebeuf et Villon », Dies Illa. Death in the Middle Ages, Liverpool, Francis Cairns, 1984, pp. 155-175 (Vinaver Studies in French, General Editors : A.R.W. James \& Jane H.M.Taylor, 1).

6. N.59, p. 371, 1. 182-186. Traduction de Roger Dubuis, (1991), p. 235.

7. N.100, p. 583, 1. 103-109. Traduction de Roger Dubuis, (1991), p. 356. 


\section{AUTEUR}

JEAN DUFOURNET

Université Paris 3 - Sorbonne Nouvelle 